\title{
Increased risk of cardiovascular disease in non-alcoholic fatty liver disease: causal effect or epiphenomenon?
}

\author{
G. Targher $\cdot$ F. Marra $\cdot$ G. Marchesini
}

Received: 13 March 2008 /Accepted: 29 July 2008 /Published online: 2 September 2008

(C) Springer-Verlag 2008

\begin{abstract}
Non-alcoholic fatty liver disease (NAFLD), comprising a spectrum of conditions ranging from pure steatosis to steatohepatitis and cirrhosis, has reached epidemic proportions and represents the most common cause of chronic liver disease in the community. The prevalence of NAFLD has been estimated to be between $20 \%$ and $30 \%$ in the general population, but this value is much higher $(\sim 70-80 \%)$ in type 2 diabetic patients, who are also at higher risk of developing advanced fibrosis and cirrhosis. Increasing recognition of the importance of NAFLD and its strong relationship with the metabolic syndrome has stimulated an interest in the possible role of NAFLD in the development of cardiovascular disease (CVD). Several epidemiological studies indicate that NAFLD, especially in its more severe forms, is linked to an increased risk of CVD, independently of underlying cardiometabolic risk factors. This suggests that NAFLD is not merely a marker of CVD, but may also be actively involved in its
\end{abstract}

Electronic supplementary material The online version of this article (doi:10.1007/s00125-008-1135-4) contains supplementary material, which is available to authorised users.

\section{G. Targher $(\square)$}

Section of Endocrinology,

Department of Biomedical and Surgical Sciences,

University of Verona,

Ospedale Civile Maggiore, Piazzale Stefani, 1,

37126 Verona, Italy

e-mail: giovanni.targher@univr.it

F. Marra

Department of Internal Medicine, Center for Research,

High Education and Transfer DENOThe,

University of Florence,

Florence, Italy

\section{G. Marchesini}

Clinical Dietetics,

Department of Internal Medicine and Gastroenterology,

University of Bologna,

Bologna, Italy pathogenesis. The possible molecular mediators linking NAFLD and CVD include the release of pro-atherogenic factors from the liver (C-reactive protein, fibrinogen, plasminogen activator inhibitor-1 and other inflammatory cytokines) as well as the contribution of NAFLD per se to whole-body insulin resistance and atherogenic dyslipidemia, in turn favouring CVD progression. The clinical impact of NAFLD on CVD risk deserves particular attention in view of the implications for screening and surveillance strategies in the growing number of patients with NAFLD.

Keywords Cardiovascular disease $\cdot$ Insulin resistance . Liver fat $\cdot$ Metabolic syndrome $\cdot$ NAFLD $\cdot$ Non-alcoholic fatty liver disease $\cdot$ Risk factors · Type 2 diabetes

\begin{tabular}{|c|c|}
\hline \multicolumn{2}{|c|}{ Abbreviations } \\
\hline CRP & C-reactive protein \\
\hline CVD & cardiovascular disease \\
\hline HOMA-IR & $\begin{array}{l}\text { homeostasis model assessment of insulin } \\
\text { resistance }\end{array}$ \\
\hline IMT & intima-media thickness \\
\hline JNK & c-Jun N-terminal kinase \\
\hline NAFLD & non-alcoholic fatty liver disease \\
\hline NASH & non-alcoholic steatohepatitis \\
\hline$N F-\kappa B$ & nuclear factor- $\mathrm{kB}$ \\
\hline PAI-1 & plasminogen activator inhibitor-1 \\
\hline
\end{tabular}

\section{Introduction}

The importance of the liver in the regulation of metabolism has been recognised for over a century and a half; several pathological conditions are associated with intra-hepatic triacylglycerol accumulation, but fatty liver has long been considered a trivial finding. It has recently been shown that fatty liver may occasionally be accompanied by necroinflammation and fibrosis, with histological lesions resembling those observed in acute alcohol intoxication. The term 
'non-alcoholic fatty liver disease' (NAFLD) is used to describe a variety of liver diseases of different severity, from pure steatosis to steatohepatitis (NASH) and cirrhosis and, rarely, hepatocellular carcinoma [1]. This metabolically derived liver disease is becoming a major problem in liver units, accounting for a huge number of consultations, day-hospital admissions for liver biopsy and, lately, admission for liver failure or transplantation [1].

The large majority of patients with NAFLD are overweight/ obese or have type 2 diabetes; another common associated clinical feature is atherogenic dyslipidaemia (i.e. high triacylglycerol, low HDL-cholesterol and increased small dense LDL-cholesterol levels). A large body of evidence suggests that NAFLD is the hepatic manifestation of the metabolic syndrome [2]. Notably, NAFLD is associated with insulin resistance in non-obese, non-diabetic individuals, suggesting that NAFLD may be an early predictor of metabolic disorders and diseases in the normal-weight population [3]. Accordingly, the cardiovascular disease (CVD) risk dictates the outcome(s) of these patients more frequently and to a greater extent than does liver disease progression. There are two key questions. (1) is NAFLD associated with CVD as a result of shared risk factors or does NAFLD per se exacerbate CVD independently of these factors? (2) Is CVD risk also present in pure steatosis, or is the necro-inflammation characteristic of NASH the necessary pro-atherogenic stimulus?

In this article we shall review the clinical and epidemiological evidence for an association between NAFLD and CVD, to promote a greater awareness for the need for comprehensive surveillance of patients with this multi-organ abnormality.

\section{Epidemiology and natural history of NAFLD}

The value reported for the prevalence of NAFLD in the community varies according to the criteria used for its assessment. Two different datasets may be considered: (1) the presence of 'bright' liver on ultrasound examination; and (2) raised serum levels of liver enzymes in individuals with no evidence of liver disease of well-defined aetiology. These two criteria do not necessarily overlap, i.e. individuals with 'bright' liver may not have elevated liver enzymes.

In the community, the prevalence of ultrasound-diagnosed NAFLD is $\sim 30 \%$, this figure being largely influenced by obesity and/or alcohol consumption [4]. If cases of excessive alcohol consumption are excluded, the prevalence is not systematically different between individuals with and without increased levels of liver enzymes [5]. When tested by proton magnetic resonance imaging, the prevalence of NAFLD is high, but again, over $60 \%$ of cases have normal serum levels of liver enzymes [6]
The use of serum liver enzymes as markers of NAFLD may produce a systematic bias. They are frequently the sole evidence of a totally asymptomatic disease, and are the most common reason for specialist consultation. The amount of liver fat is proportional to the number of features of the metabolic syndrome and is closely correlated with serum liver enzyme levels [7], which are subject to large inter-individual variability. On average, patients with NAFLD are more likely to have excess intraabdominal visceral fat and inflammatory changes in adipose tissue [8]; fat accumulation in the liver rather than in skeletal muscle is associated with features of the metabolic syndrome [9].

Metabolically induced liver disease may also act as a contributing factor in the progression of liver disease of a different aetiology $[5,10]$. Because of the high prevalence of metabolic diseases in the adult population, there is a frequent chance association, and there is evidence that the progression of viral or genetic liver diseases may be more severe in individuals with steatosis, leading to an underestimation of the overall clinical impact of NAFLD on morbidity and mortality $[5,10]$.

The natural history of NAFLD remains largely undefined. Population and cohort-based studies suggested that the regular course is relatively benign in pure steatosis $[10,11]$, with a long-term prognosis similar to that for the general population [11]. However, a few clinical series observed that hepatic steatosis is a risk factor for NAFLD progression, mainly in the presence of obesity and type 2 diabetes [12]. Liver fat content is $\sim 80 \%$ higher in type 2 diabetic patients than in age-, sex- and BMImatched non-diabetic patients with NAFLD, and their serum liver enzymes are not as representative of the severity of intra-hepatic fat accumulation [13]. These data explain why type 2 diabetes is a risk factor for NAFLD progression, and are consistent with studies reporting a high standardised mortality ratio for liver disease in type 2 diabetes [14].

NAFLD progression may eventually result in hepatocellular carcinoma [12], the incidence of which is lower than expected on the basis of the prevalence of the underlying cryptogenic cirrhosis, possibly as a result of slow disease progression [15] and CVD-related mortality.

\section{Evidence linking NAFLD with CVD}

The main cross-sectional [16-31] and prospective [11, 3243] studies on the association between NAFLD and intermediate markers of CVDor clinical outcomes are shown in Tables 1 and 2 of the Electronic supplementary material. 
NAFLD, endothelial dysfunction, early carotid atherosclerosis and left ventricular dysfunction

NAFLD is associated with circulatory endothelial dysfunction $[16,17]$, a marker of early atherosclerosis. Compared with non-steatotic controls, non-diabetic patients with NAFLD show a significant decrease in brachial artery flow-mediated vasodilation, which is related to the severity of NAFLD histology, independently of age, sex, BMI and HOMA-IR [16]. Moreover the same study reported that the 10 year probability of CVD events (calculated using the Framingham risk score) is moderately increased in NAFLD, particularly in NASH [16]. Similarly, in patients with metabolically well-controlled type 2 diabetes, mildly elevated levels of serum liver enzymes are associated with abnormal brachial flow-mediated vasodilation, independently of whole body insulin sensitivity, as measured by the euglycaemic-hyperinsulinaemic clamp method, and other prognostic factors [17].

Carotid artery intima-media thickness (IMT), another marker of early atherosclerosis, is also markedly increased in patients with NAFLD patients compared with that in age-, sex- and BMI-matched healthy controls [20-25], and NAFLD histology predicts carotid IMT independently of traditional risk factors, HOMA-IR and features of the metabolic syndrome [24]. Similarly, patients with NAFLD, also in the absence of obesity, hypertension and diabetes, exhibit echocardiographic features of early left ventricular dysfunction [18] and impaired energy metabolism as measured by cardiac ${ }^{31} \mathrm{P}$ magnetic resonance spectroscopy [19].

These studies provide strong evidence for an increased risk of CVD in patients with NAFLD, and most of these patients are expected to develop CVD events in the long term. Obviously, they also raise the question of whether CVD events will occur prior to the development of advanced liver disease and whether aggressive treatment of underlying CVD risk factors is an important treatment modality for all patients with NAFLD.

\section{NAFLD and increased prevalence of clinical CVD}

In both non-diabetic and type 2 diabetic individuals, those with ultrasound-diagnosed NAFLD have a higher prevalence of clinical CVD than do their non-steatotic counterparts, and this association is independent of obesity and traditional risk factors $[26,29]$. In patients consecutively submitted to undergo elective coronary angiography, NAFLD was correlated with a greater severity of coronary artery disease, independently of traditional risk factors and components of the metabolic syndrome [30]. Moreover, fatty liver is independently associated with lower insulin- stimulated myocardial glucose uptake and reduced coronary functional capacity in type 2 diabetic patients with known CVD [27]. Finally, a recent autopsy study of 742 children found that the prevalence of coronary heart disease is twofold higher in those with NAFLD [31].

These studies further suggest that NAFLD patients are at a higher risk of CVD. However, the cross-sectional nature of the studies calls for caution in interpreting the data, and large prospective studies are needed to assess whether CVD risk affects long-term survival.

\section{NAFLD and increased incidence of CVD}

Overall, NAFLD patients have a higher risk of all-cause death than the general population, mainly due to CVD or liver-related causes. In a community-based NAFLD cohort followed for 7.6 years, the rate of all-cause mortality, most commonly due to malignancy and CVD, was significantly higher than in the general population [35], and mortality rates from CVD were the second most common cause of death in 132 patients with biopsy-proven NAFLD followed for 18 years, with rates equalling those of liver-related deaths [33]. Furthermore, the 14 year risk of CVD mortality was doubled in 129 patients with biopsy-proven NAFLD and elevated liver enzymes compared with that in the general population [38].

These studies examining the natural history of NAFLD are limited by the relatively small number of patients and by the fact that they originate from tertiary specialist centres, thus limiting their external validity. However, a strong association between elevated levels of serum liver enzymes and increased CVD risk has been reported by numerous population-based studies [32, 36, 39-41]. For example, in a study of 163,944 middle-aged participants, mildly elevated levels of liver enzymes were independently associated with an increased CVD mortality rate in both sexes [36]. These results have been confirmed in the Hoorn Study [40] and in the Framingham Heart Study [41], both of which reported that raised serum levels of liver enzymes predicted an increased risk of CVD, independently of traditional risk factors and components of the metabolic syndrome.

In the Valpolicella Heart Diabetes Study, the presence of ultrasound-diagnosed NAFLD in a large cohort of type 2 diabetic individuals was associated with an increased incidence of CVD events, independently of a broad number of confounding factors [37, 42]. Almost identical results were reported in a community-based cohort of 1,637 nondiabetic individuals [43].

Notably, these studies indicate that NAFLD is associated with an increased risk of CVD, independently of 
several established risk factors, thus suggesting that NAFLD might confer an excess risk over and above what would be expected to be associated with the underlying CVD risk factors. Patients with NAFLD should be candidates not only for aggressive treatment of their liver disease, but also for aggressive treatment of underlying risk factors, as many patients with NAFLD will have major CVD events and die prior to the development of advanced liver disease.

\section{Biological mechanisms potentially responsible for accelerated atherogenesis in NAFLD}

Current understanding of the pathogenesis of NAFLD implies that lipids accumulate in hepatocytes, mainly in the form of triacylglycerol, in the presence of insulin resistance. The close inter-relationships between NAFLD, insulin resistance and the metabolic syndrome make it extremely difficult to dissect any cause-effect relationship leading to the increased CVD risk. The biological mechanisms potentially responsible for accelerated atherogenesis in NAFLD may either have origins in the visceral adipose tissue or in the liver, or have the liver as the target of systemic abnormalities, with both sites expanding the defects (as shown schematically in Fig. 1). A leading role in the development of insulin resistance, inflammation and NAFLD is likely to be played by excess adiposity, including ectopic fat deposition - principally in abdominal depots but also in the heart, skeletal muscle and other organs. In fact, ectopic fat deposition is a source of multiple factors involved in atherogenesis, such as NEFA, hormones, pro-inflammatory cytokines and adipocytokines [44].

Intra-hepatic triacylglycerol accumulation (fatty liver) is the result of an increased flux of NEFA mainly derived from the lipolysis of expanded abdominal adipose tissue, which occurs in response to insulin resistance, but also from dietary chylomicrons and de novo intrahepatic lipogenesis $[1,45]$. In turn, NAFLD, especially in its necro-inflammatory form (NASH), may cause further increases in whole body insulin resistance and promote systemic inflammation through the release of pro-inflammatory and pro-atherogenic factors [44-46]. Indeed, liver fat content in non-diabetic obese individuals is the best predictor of insulin action in the liver, skeletal muscle and adipose tissue, independent of percentage body fat [47].

Patients with the metabolic syndrome typically have atherogenic dyslipidaemia, which is characterised by high triacylglycerol, low HDL-cholesterol, increased small dense LDL particles, and increased apolipoprotein B100 concentration. The pivotal abnormality is an increased rate of hepatic triacylglycerol synthesis and VLDL particle production, which results in secondary abnormalities characterised by low HDL-cholesterol levels and increased LDL particle number and density [44, 45, 48]. As mentioned above, insulin resistance is regarded as a major driving force for atherogenic dyslipidaemia, because it increases NEFA release, which stimulates hepatic triacylglycerol
Fig. 1 Biological mechanisms potentially responsible for accelerated atherogenesis in NAFLD

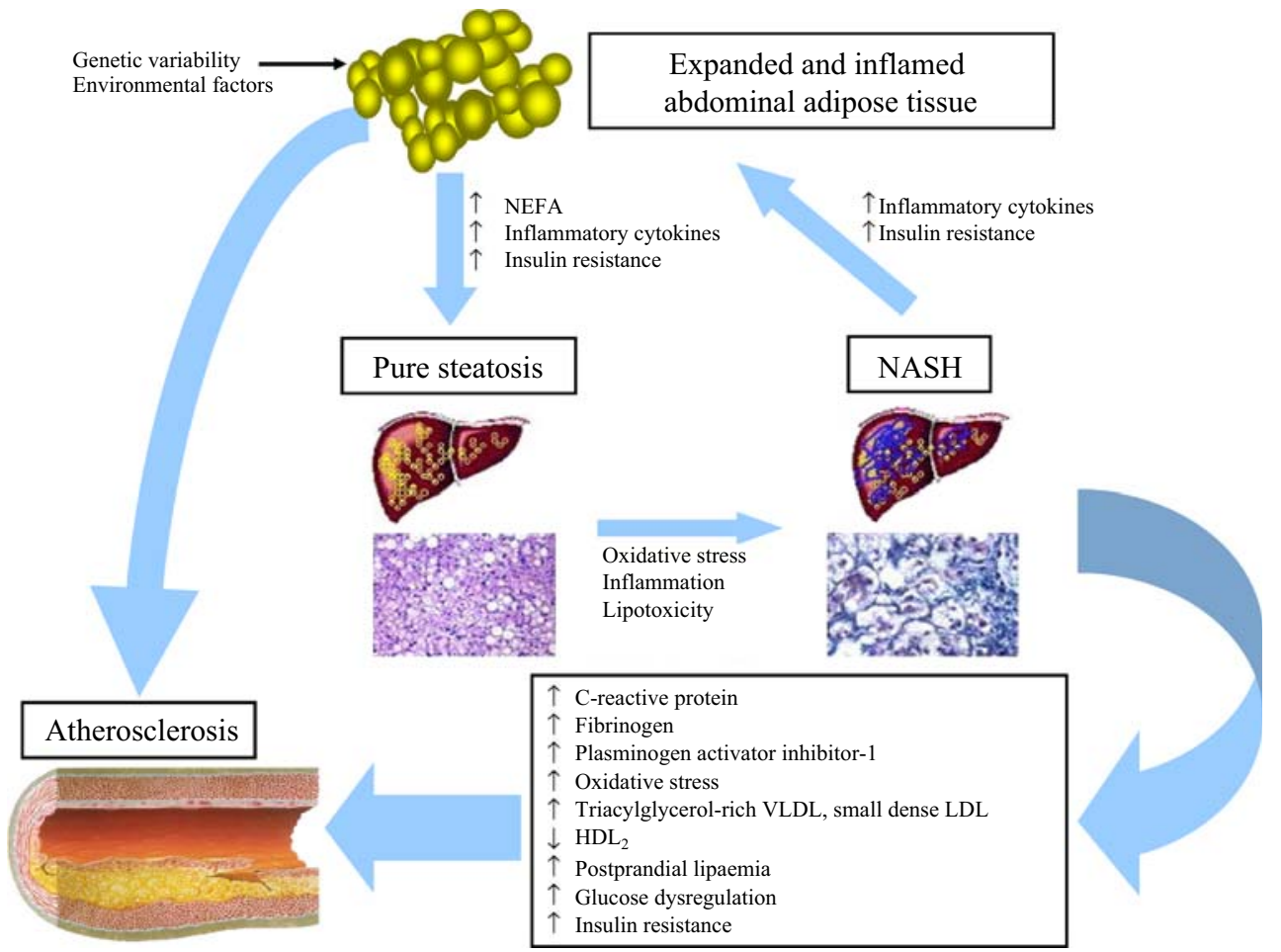


output $[44,45,48]$. Increased lipid availability within the liver and insulin resistance also inhibit apolipoprotein B degradation, stabilising the formation of more VLDL [45, 48]. The ability of insulin infusion to suppress VLDL1 secretion is impaired in individuals with high vs low liver fat content, resulting in VLDL1 overproduction [45]. Accordingly, it has been shown that hepatic steatosis in type 2 diabetes is a better predictor of the severity and composition of dyslipidaemia than are hyperglycaemia or insulin resistance as measured by the euglycaemic-hyperinsulinaemic clamp [49]. Liver fat content is a close correlate of postprandial lipaemia and increased levels of oxidised LDL after an oral fat load, much better than measures of glucose metabolism or body adiposity $[50,51]$.

Parallel to changes in metabolic substrates, chronic inflammation is causally involved in insulin resistance and the metabolic syndrome, as mainly demonstrated by mechanistic studies in animal models [52]. Ectopic fat deposition in visceral adipose depots, heart and other depots increases the expression of several pro-inflammatory mediators such as monocyte chemotactic protein-1 and IL-6, leading to local macrophage infiltration and associated systemic chronic inflammation [8, 44, 45, 53, 54]. Specifically, recent human data suggest that abdominal obesity is associated with increased levels of circulating IL-6 [53, 54]. Adipose tissue inflammation is one of the earliest steps in the chain of events leading to whole body insulin resistance [52]. Activation of pro-inflammatory pathways is mediated by cytokine receptors and pattern recognition receptors, including toll-like receptor and receptors for advanced glycation endproducts [44, 52]. These pathways converge on two main intracellular signalling pathways, namely, nuclear factor- $\mathrm{kB}(\mathrm{NF}-\mathrm{kB})$ and c-Jun N-terminal kinase (JNK). JNK contributes to insulin resistance via direct phosphorylation and degradation of IRS1, dampening the intracellular signalling pathway downstream of the insulin receptor [52]. NF-KB activation has been demonstrated in the liver of patients with NASH [55], and leads to increased transcription of numerous proinflammatory genes that amplify the response [52]. In the presence of systemic inflammation, the liver is again both the target of and contributor to inflammatory changes. Hepatic steatosis is associated with increased production of IL-6 and other pro-inflammatory cytokines by hepatocytes and non-parenchymal cells, including Kupffer cells and hepatic stellate cells. Increased intra-hepatic cytokine expression results from local NF-KB activation, mediated by hepatocellular damage and fat-derived factors [46, 56, 57], and is likely to play a major role in NAFLD progression $[1,46,56]$ and CVD pathogenesis. Along these lines, an atherogenic role of liver inflammation is supported by the observation that CVD risk is greater in NASH than in pure steatosis $[33,34,38]$, and it is strongly associated with elevated serum liver enzymes, a surrogate marker of liver necro-inflammation [32, 36, 39-41]. Consistent with the hypothesis that liver inflammation plays a role in the pathogenesis of CVD, we have also demonstrated that both patients with NASH and those with chronic viral hepatitis B and C have markedly greater carotid IMT values than do healthy controls [58].

Another effect of adipose tissue expansion and inflammation is an imbalance in the secretion of different adipokines, which could contribute to the NAFLD progression and the resulting increase in CVD risk [46, 56]. Patients with NAFLD exhibit reduced adiponectin levels, which are inversely correlated with the severity of NAFLD histology [59]. The reduced production of adiponectin associated with obesity may contribute to the progression of NAFLD [46, 56, 59] and CVD [60], since this hormone appears to exert strong anti-inflammatory and anti-fibrotic effects. In contrast, leptin levels are increased in NAFLD, and may play a role in the progression from pure steatosis to NASH, at least according to studies in animal models $[46,56]$.

The liver is central for the production of classical biomarkers of inflammation and endothelial dysfunction, the secretion of which partly depends on factors that are upregulated in the presence of insulin resistance and the metabolic syndrome. IL- 6 and TNF- $\alpha$ are the major stimuli responsible for increased hepatic production of C-reactive protein (CRP), fibrinogen and other acute-phase proteins $[44,52]$. It has been shown that fibrinogen and CRP levels, which are known CVD risk factors, are increased in NAFLD patients, particularly in those with NASH [61, 62]. The liver is also an important site of production of plasminogen activator inhibitor-1 (PAI-1), another known risk factor for CVD [44]. Plasma PAI-1 concentrations are remarkably elevated in NAFLD patients, and correlate with the histological severity of NAFLD independently of traditional risk factors, HOMA-IR and components of the metabolic syndrome [62].

\section{Conclusions}

The introductory key questions were whether NAFLD plays a direct role in CVD pathogenesis and whether an inflamed liver (NASH) adds to the CVD risk above the effect of steatosis alone. The answer is affirmative: current evidence indicates a strong, graded relationship between the histological severity of NAFLD and atherosclerosis, but NAFLD remains associated with an increased CVD risk after adjustment for traditional risk factors and components of the metabolic syndrome. The biological link may be the shared metabolic and prothrombotic inflammatory factors and the common molecular events playing a role in the pathogenesis of NAFLD and CVD. However, there is now 
growing evidence that NAFLD/NASH per se can exacerbate CVD risk, independently of shared metabolic risk factors, through the release of pro-atherogenic factors.

NAFLD and CVD share similar treatment strategies aimed primarily at improving insulin resistance and modifying the cardiometabolic risk factors. Pharmacotherapy should probably be reserved for those patients at highest risk of complications, i.e. those with NASH, type 2 diabetes, dyslipidaemia and obesity. The lack of adequately powered randomised controlled trials of sufficient duration and with histological end-points makes definitive recommendations difficult at present [1]. Current treatment recommendations are limited to gradual weight reduction, regular exercise and to the tight control of each component of the metabolic syndrome. Insulin-sensitising agents (metformin and glitazones) also seem to be effective in the treatment of NAFLD [1]; however, it is not known whether improving NAFLD will ultimately prevent the development and progression of CVD. From a clinical practice standpoint, it is crucial that specialists and practising clinicians be aware of the strong association between NAFLD and increased CVD risk, given the growing number of patients with NAFLD in the community. Hepatic steatosis is an occasional finding; this should prompt clinicians to define underlying CVD risk factors, which warrant evaluation and treatment as much as liver disease itself. Liver disease surveillance is necessary, given the long-term risk of advanced disease, but tight control of underlying CVD risk factors also remains essential.

Acknowledgements The authors thank C. P. Day (Institute of Cellular Medicine, The Medical School, Newcastle University, Newcastle upon Tyne, UK) for his helpful comments on the final version of the manuscript.

Duality of interest The authors declare that there is no duality of interest associated with this manuscript.

\section{References}

1. Adams LA, Lindor KD (2007) Nonalcoholic fatty liver disease. Ann Epidemiol 17:863-869

2. Marchesini G, Bugianesi E, Forlani G et al (2003) Nonalcoholic fatty liver, steatohepatitis, and the metabolic syndrome. Hepatology 37:917-923

3. Kim HJ, Lee KE, Kim DJ et al (2004) Metabolic significance of nonalcoholic fatty liver disease in nonobese, nondiabetic adults. Arch Intern Med 164:2169-2175

4. Bellentani S, Saccoccio G, Masutti F et al (2000) Prevalence of and risk factors for hepatic steatosis in Northern Italy. Ann Intern Med 132:112-117

5. Bedogni G, Miglioli L, Masutti F, Tiribelli C, Marchesini G, Bellentani S (2005) Prevalence of and risk factors for nonalcoholic fatty liver disease: the Dionysos nutrition and liver study. Hepatology 42:44-52
6. Browning JD, Szczepaniak LS, Dobbins R et al (2004) Prevalence of hepatic steatosis in an urban population in the United States: impact of ethnicity. Hepatology 40:1387-1395

7. Kotronen A, Westerbacka J, Bergholm R, Pietilainen KH, Yki-Jarvinen $\mathrm{H}$ (2007) Liver fat in the metabolic syndrome. J Clin Endocrinol Metab 92:3490-3497

8. Kolak M, Westerbacka J, Velagapudi VR et al (2007) Adipose tissue inflammation and increased ceramide content characterize subjects with high liver fat content independent of obesity. Diabetes 56:1960-1968

9. Kotronen A, Seppala-Lindroos A, Bergholm R, Yki-Jarvinen H (2008) Tissue specificity of insulin resistance in humans: fat in the liver rather than muscle is associated with features of the metabolic syndrome. Diabetologia 51:130-138

10. Bedogni G, Miglioli L, Masutti F et al (2007) Incidence and natural course of fatty liver in the general population: the Dionysos study. Hepatology 46:1387-1391

11. Dam-Larsen S, Franzmann M, Andersen IB et al (2004) Long term prognosis of fatty liver: risk of chronic liver disease and death. Gut 53:750-755

12. Marchesini G, Forlani G, Bugianesi E (2005) Is liver disease a threat to patients with metabolic disorders? Ann Med 37:333-346

13. Kotronen A, Juurinen L, Hakkarainen A et al (2008) Liver fat is increased in type 2 diabetic patients and underestimated by serum alanine aminotransferase compared with equally obese nondiabetic subjects. Diabetes Care 31:165-169

14. de Marco R, Locatelli F, Zoppini G, Verlato G, Bonora E, Muggeo M (1999) Cause-specific mortality in type 2 diabetes. The Verona Diabetes Study. Diabetes Care 22:756-761

15. Poynard T, Mathurin P, Lai CL et al (2003) A comparison of fibrosis progression in chronic liver diseases. J Hepatol 38:257-265

16. Villanova N, Moscatiello S, Ramilli S et al (2005) Endothelial dysfunction and cardiovascular risk profile in nonalcoholic fatty liver disease. Hepatology 42:473-480

17. Schindhelm RK, Diamant M, Bakker SJ et al (2005) Liver alanine aminotransferase, insulin resistance and endothelial dysfunction in normotriglyceridaemic subjects with type 2 diabetes mellitus. Eur J Clin Invest 35:369-374

18. Goland S, Shimoni S, Zornitzki T et al (2006) Cardiac abnormalities as a new manifestation of nonalcoholic fatty liver disease: echocardiographic and tissue Doppler imaging assessment. J Clin Gastroenterol 40:949-955

19. Perseghin G, Lattuada G, De Cobelli F et al (2008) Increased mediastinal fat and impaired left ventricular energy metabolism in young men with newly found fatty liver. Hepatology 47:51-58

20. Targher G, Bertolini L, Padovani R, Zenari L, Zoppini G, Falezza G (2004) Relation of nonalcoholic hepatic steatosis to early carotid atherosclerosis in healthy men: role of visceral fat accumulation. Diabetes Care 27:2498-2500

21. Brea A, Mosquera D, Martin E, Arizti A, Cordero JL, Ros E (2005) Nonalcoholic fatty liver disease is associated with carotid atherosclerosis. A case-control study. Arterioscler Thromb Vasc Biol 25:1045-1050

22. Targher G, Bertolini L, Padovani R et al (2006) Non-alcoholic fatty liver disease is associated with carotid artery wall thickness in diet-controlled type 2 diabetic patients. J Endocrinol Invest 29:55-60

23. Volzke H, Robinson DM, Kleine V et al (2005) Hepatic steatosis is associated with an increased risk of carotid atherosclerosis. World J Gastroenterol 11:1848-1853

24. Targher G, Bertolini L, Padovani R et al (2006) Relations between carotid artery wall thickness and liver histology in subjects with nonalcoholic fatty liver disease. Diabetes Care 29:1325-1330

25. Fracanzani AL, Burdick L, Raselli S et al (2008) Carotid artery intima-media thickness in nonalcoholic fatty liver disease. Am J Med 121:72-78 
26. Lin YC, Lo HM, Chen JD (2005) Sonographic fatty liver, overweight and ischemic heart disease. World J Gastroenterol 11:4838-4842

27. Lautamäki R, Borra R, Iozzo $P$ et al (2006) Liver steatosis coexists with myocardial insulin resistance and coronary dysfunction in patients with type 2 diabetes. Am J Physiol Endocrinol Metab 291:E282-E290

28. Ioannou GN, Weiss NS, Boyko EJ, Mozaffarian D, Lee SP (2006) Elevated serum alanine aminotransferase activity and calculated risk of coronary heart disease in the United States. Hepatology 43:1145-1151

29. Targher G, Bertolini L, Padovani R et al (2007) Prevalence of nonalcoholic fatty liver disease and its association with cardiovascular disease among type 2 diabetic patients. Diabetes Care 30:1212-1218

30. Mirbagheri SA, Rashidi A, Abdi S, Saedi D, Abouzari M (2007) Liver: an alarm for the heart? Liver Int 27:891-894

31. Schwimmer JB, Deutsch R, Behling C, Lavine JE (2005) Fatty liver as a determinant of atherosclerosis. Hepatology 42:610A, [Abstract]

32. Wannamethee G, Ebrahim S, Shaper AG (1995) Gammaglutamyltransferase: determinants and association with mortality from ischemic heart disease and all causes. Am J Epidemiol 142:699-708

33. Matteoni CA, Younossi ZM, Gramlich T, Boparai N, Liu YC, McCullough AJ (1999) Nonalcoholic fatty liver disease: a spectrum of clinical and pathological severity. Gastroenterology 116:1413-1419

34. Jepsen P, Vilstrup H, Mellemkjaer L et al (2003) Prognosis of patients with a diagnosis of fatty liver: a registry-based cohort study. Hepatogastroenterology 50:2101-2104

35. Adams LA, Lymp JF, St Sauver J et al (2005) The natural history of nonalcoholic fatty liver disease: a population-based cohort study. Gastroenterology 129:113-121

36. Ruttmann E, Brant LJ, Concin H, Diem G, Rapp K, Ulmer H (2005) g-Glutamyltransferase as a risk factor for cardiovascular disease mortality: an epidemiological investigation in a cohort of 163,944 Austrian adults. Circulation 112:2130-2137

37. Targher G, Bertolini L, Poli F et al (2005) Nonalcoholic fatty liver disease and risk of future cardiovascular events among type 2 diabetic patients. Diabetes 54:3541-3546

38. Ekstedt M, Franzen LE, Mathiesen UL et al (2006) Long-term follow-up of patients with NAFLD and elevated liver enzymes. Hepatology 44:865-873

39. Lee DH, Silventoinen K, Hu G et al (2006) Serum gammaglutamyltransferase predicts non-fatal myocardial infarction and fatal coronary heart disease among 28,838 middle-aged men and women. Eur Heart J 27:2170-2176

40. Schindhelm RK, Dekker JM, Nijpels G et al (2007) Alanine aminotransferase predicts coronary heart disease events: a 10-year follow-up of the Hoorn Study. Atherosclerosis 191:391-396

41. Lee DS, Evans JC, Robins SJ et al (2007) Gamma glutamyltransferase and metabolic syndrome, cardiovascular disease, and mortality risk: the Framingham Heart Study. Arterioscler Thromb Vasc Biol 27:127-133

42. Targher G, Bertolini L, Rodella S et al (2007) Nonalcoholic fatty liver disease is independently associated with an increased incidence of cardiovascular events in type 2 diabetic patients. Diabetes Care 30:2119-2121

43. Hamaguchi M, Kojima T, Takeda N et al (2007) Nonalcoholic fatty liver disease is a novel predictor of cardiovascular disease. World J Gastroenterol 13:1579-1584
44. Van Gaal LF, Mertens IL, De Block CE (2006) Mechanisms linking obesity with cardiovascular disease. Nature 444:875-880

45. Adiels M, Taskinen MR, Borén J (2008) Fatty liver, insulin resistance, and dyslipidemia. Curr Diab Rep 8:60-64

46. Day CP (2006) From fat to inflammation. Gastroenterology 130:207-210

47. Korenblat KM, Fabbrini E, Mohammed BS, Klein S (2008) Liver, muscle, and adipose tissue insulin action is directly related to intrahepatic triglyceride content in obese subjects. Gastroenterology 134:1369-1375

48. Ginsberg HN (2006) Review: Efficacy and mechanisms of action of statins in the treatment of diabetic dyslipidemia. J Clin Endocrinol Metab 91:383-392

49. Toledo FG, Sniderman AD, Kelley DE (2006) Influence of hepatic steatosis (fatty liver) on severity and composition of dyslipidemia in type 2 diabetes. Diabetes Care 29:1845-1850

50. Matikainen N, Mänttäri S, Westerbacka J et al (2007) Postprandial lipemia associates with liver fat content. J Clin Endocrinol Metab 92:3052-3059

51. Gambino R, Cassader M, Pagano G, Durazzo M, Musso G (2007) Polymorphism in microsomal triglyceride transfer protein: a link between liver disease and atherogenic postprandial lipid profile in NASH? Hepatology 45:1097-1107

52. Shoelson SE, Lee J, Goldfine AB (2006) Inflammation and insulin resistance. J Clin Invest 116:1793-1801

53. Fontana L, Eagon JC, Trujillo ME, Scherer PE, Klein S (2007) Visceral fat adipokine secretion is associated with systemic inflammation in obese humans. Diabetes 56:1010-1013

54. Cartier A, Lemieux I, Alméras N, Tremblay A, Bergeron J, Despres JP (2008) Visceral obesity and plasma glucose-insulin homeostasis: contributions of interleukin- 6 and tumor necrosis factor-alpha in men. J Clin Endocrinol Metab 93:1931-1938

55. Ribeiro PS, Cortez-Pinto H, Solà S et al (2004) Hepatocyte apoptosis, expression of death receptors, and activation of NF-kB in the liver of nonalcoholic and alcoholic steatohepatitis patients. Am J Gastroenterol 99:1708-1717

56. Marra F, Gastaldelli A, Svegliati Baroni G, Tell G, Tiribelli C (2008) Molecular basis and mechanisms of progression of nonalcoholic steatohepatitis. Trends Mol Med 14:72-81

57. Wieckowska A, Papouchado BG, Li Z, Lopez R, Zein NN, Feldstein AE (2008) Increased hepatic and circulating interleukin-6 levels in human nonalcoholic steatohepatitis. Am J Gastroenterol 103:13721379

58. Targher G, Bertolini L, Padovani R et al (2007) Differences and similarities in early atherosclerosis between patients with nonalcoholic steatohepatitis and chronic hepatitis B and C. J Hepatol 46:1126-1132

59. Hui JM, Hodge A, Farrell GC, Kench JG, Kriketos A, George J (2004) Beyond insulin resistance in NASH: TNF-alpha or adiponectin? Hepatology 40:46-54

60. Matsuzawa Y, Funahashi T, Kihara S, Shimomura I (2004) Adiponectin and metabolic syndrome. Arterioscler Thromb Vasc Biol 24:29-33

61. Yoneda M, Mawatari H, Fujita K, Iida H et al (2007) Highsensitivity C-reactive protein is an independent clinical feature of nonalcoholic steatohepatitis (NASH) and also of the severity of fibrosis in NASH. J Gastroenterol 42:573-582

62. Targher G, Bertolini L, Rodella S et al (2008) NASH predicts plasma inflammatory biomarkers independently of visceral fat in men. Obesity (Silver Spring) 16:1394-1399 